\title{
Circuit
}

Musiques contemporaines

\section{Électroacoustique et perspective phonoculturelle}

\section{Alain Savouret}

Volume 13, numéro 1, 2002

L'électroacoustique : à la croisée des chemins?

URI : https://id.erudit.org/iderudit/902260ar

DOI : https://doi.org/10.7202/902260ar

Aller au sommaire du numéro

\section{Éditeur(s)}

Les Presses de l'Université de Montréal

\section{ISSN}

1183-1693 (imprimé)

1488-9692 (numérique)

Découvrir la revue

\section{Citer cet article}

Savouret, A. (2002). Électroacoustique et perspective phonoculturelle. Circuit, 13(1), 9-20. https://doi.org/10.7202/902260ar

\section{Résumé de l'article}

L'électroacoustique, comprise uniquement comme un " genre » de musique contemporaine supplémentaire, réduit l'importance de la régénération de notre « entendement » du monde audible que l'électroacoustique en général (fixation/mémorisation et diffusion de traces sonores) a produit depuis le milieu du $\mathrm{XX}^{\mathrm{e}}$ siècle.

Enfin une représentation du monde audible de chacun était possible, si longtemps après la représentation du monde visible que faisaient nos lointains ancêtres sur les parois des grottes. Représentation comprise comme persistance d'un " ancien " moment présent vécu par le « traceur " de l'époque. Paradoxalement, plutôt que de se placer dans une large perspective phonoculturelle et « se jouer » des réalités sonores quotidiennes, ce qui semblait une aventure nouvelle et surprenante, les courants électroacoustiques les plus influents ont cherché assez vite à rejoindre la musique instrumentale en ce qu'elle est plutôt une "représentation ". Ajoutons à cette tendance une sorte de méfiance vis-à-vis des sons « de référence » au profit des sons « originaux » (synthèse, transmutation...) qui facilitent une possible écriture, un « langage de traces » (et donc un savoir), contrairement à une démarche « aurale » où la mémorisation de traces (ici mécaniques) peut créer une relation im-médiate (sans intermédiaire) des hommes à leur monde audible. L'électroacoustique n'a pas à choisir un chemin mais simplement à ne pas oublier sa pertinente originalité.
Ce document est protégé par la loi sur le droit d'auteur. L’utilisation des services d'Érudit (y compris la reproduction) est assujettie à sa politique d'utilisation que vous pouvez consulter en ligne.

https://apropos.erudit.org/fr/usagers/politique-dutilisation/ 


\title{
Électroacoustique et perspective phonoculturelle
}

\author{
Alain Savouret
}

Les propos tenus dans cette communication devront être considérés comme issus d'une humble et partielle réflexion expérimentale, ne serait-ce que pour réintroduire un qualificatif qui sonne si bien quand on le pose à côté de musique, s'en faisant la résonance logique et pertinente dès que l'on conçoit l'art comme une remise en question permanente de nos usages.

\section{Musique électroacoustique ou électroacoustique?}

Il y a une aubaine dans le titre générique "L'électroacoustique : à la croisée des chemins?", une aubaine que je ne vais pas manquer d'exploiter. Dans ce titre, La musique électroacoustique est certes sous-entendue, mais je profiterai du sousentendu pour jouer au mal-entendant et distinguer, pour ma communication, l'électroacoustique de la musique électroacoustique; distinguer le phénomène global lié à l'intrusion de la captation du son et de sa diffusion dans notre relation perceptive au monde et l'exercice esthétique de cette technique dans le cadre de l'art musical contemporain.

Opter, dans un premier temps, pour électroacoustique ainsi définie, c'est se situer en amont d'un genre de musique (la musique électroacoustique) qui a plus ou moins bien pris sa place dans l'art musical selon les époques, les continents, les sociétés, les usages, etc. C'est placer l'électroacoustique dans le cadre plus élémentaire et plus vaste de ce que j'appelle la phonoculture, culture du son donc, où comme pour la culture de la terre (l'agriculture...) les angles d'approche du sujet sont nombreux et variés, aigus ou obtus, et qu'il faut bien se limiter.

la musique électroacoustique, composante particulière de l'électroacoustique, s'inscrit, à l'instar de toutes les musiques, dans ce cadre très général à l'intérieur 
duquel pourraient se placer aussi toutes sortes d'approches du sonore : scientifique, médicale, industrielle, écologique, etc. Dans l'état actuel de mes réflexions pratiques/pratiquées là la fois commodes, faciles à utiliser pour mieux trier... et expérimentées de diverses façons...), placer la musique (donc toutes les...) dans une perspective phonoculturelle, c'est aborder le son sous quatre angles, quatre éclairages différents : la textualité du son (relation du son au signe, le son écrit), la matérialité du son (formes et matière inventées au XX' siècle, pour lesquelles l'électroacoustique est historiquement déterminante), la contextualité du son (topicité, chronicité et socialité donneuses de sens au son), enfin l'auralité du son'. Ces différents éclairages seront tour à tour utilisés en cours de discours.

Revenons à cette distinction entre électroacoustique et musique électroacoustique; elle est motivée par le refus théorique de penser la musique électroacoustique uniquement comme un genre de musique contemporaine greffé à la tradition instrumentale ou cohabitant avec celle-ci, avec des réussites diverses sous la forme dite mixte. Refus de la penser comme une suite, un prolongement filial de l'univers instrumental, une sorte de modernité ajoutée. Quitte à être l'engendrée de quelque chose, autant l'être du cinéma, un art antérieur lui aussi en appui technique sur la fixation de traces sur un support déroulant ${ }^{2}$.

Bien souvent l'électroacoustique ${ }^{3}$ reste une activité annexe tenue à distance correcte par le corps enseignant majoritaire non directement concerné. On lui refuse encore le pouvoir fondamental de régénérer notre entendement du musical, toutes époques confondues; la capacité inventive de l'instrumentiste ou du compositeur ; les modes d'accès éducatifs à la pratique musicale pour les enfants mais aussi pour ceux de plus en plus nombreux qui ne veulent pas vivre la musique par procuration, en consommateurs disciplinés. L'électroacoustique comprise en ce sens est loin d'être dans l'impasse.

Certes, la cohabitation instrument/haut-parleur à travers la musique mixte écrite reste le métissage toléré dans les institutions; "c'est toujours cela de gagné", dirons-nous. J'ai bien parlé de musique mixte écrite pour la distinguer de tout un courant musical actuellement très actif en France, lié à l'improvisation instrumentale non idiomatique en appui sur tous les types de contexte, par exemple l'environnement naturel (un lieu) ou celui, virtuel, qu'une phonographie ${ }^{4}$ diffusée par hautparleurs peut générer ${ }^{5}$.

Par ailleurs, ce n'est pas la seule cohabitation amorcée depuis le milieu du XXe siècle : la danse, le cinéma d'art et d'essai, le théâtre, les galeries et des formes d'expression plus transversales ont accueilli cette technique de réalisation avec chaleur et appétit. La musique inscrite électroacoustique a depuis longtemps trouvé sa place aux côtés de la musique écrite instrumentale pour ce qui concerne ces demandes en mariages de toutes sortes.

Cette musique inscrite s'est depuis "art-visualisée ", "techno-popisée", "homestudiotisée » à qui mieux mieux et personne ne s'en plaindra; ce ne sont que des
1. Relation immédiate de l'énergie humaine au son dès lors que l'oreille - auris - est maître du jeu, c'est-à-dire aussi maître du je-ici-maintenant.

2. Exemple de malentendu : si elle est présente institutionnellement dans certaines écoles de musique agréées par l'État en France lavec des professeurs titulaires de diplômes d'État), je ne suis pas certain que dans tous ces lieux de formation musicale spécialisée les décideurs d'orientations comprennent l'électroacoustique comme la chance de former l'oreille des élèves au sonore généralisé et ce, au-delà même de toutes cultures musicales, comprennent l'électroacoustique comme une sorte possible de solfège pratique $d u X X$ siècle sonore, venant épauler le solfège usuel à destination instrumentale, qui s'essouffle dès que les sons deviennent hors de portée lje renvoie à mon article "Solfège de code ou solfège de l'entendre : quelle formation de l'oreille?", dans Le Conservatoire de Paris - Deux cents ans de pédagogie, Buchet/Chastel, 1999).

3. Musique ou pas, ou avec réserve, ou avec malentendu..

4. Définissons la phonographie comme l'enregistrement d'un moment de vie dans lequel est décelable, comme pour la tragédie française, une unité de lieu, de temps et d'action; plutôt que d'une prise de son, parlons d'une prise de sens et même de la fixation temporelle d'une relation entre le preneur de son/sens et l'objet de sa captation. II ne s'agit en aucun cas, par exemple, d'un montage de sons anecdotiques divers ou de l'insertion fugitive de sources sonores identifiables dans une phrase ou séquence composée d'éléments de morpho-typologies complexes et variées. 5. Je fais une rapide allusion au travail effectué, entre autres, par Jean Pallandre et Marc Pichelin dans le cadre de "Ouie Dire Production »/GMEAlbi. 
variations de genre de la permanence électroacoustique, si l'on veut utiliser des notions schaefferienne.

Et il faudrait avoir une vision et une conception bien intégristes de la musique électroacoustique pour lui refuser ces allers et retours forcément régénérant, même et y compris dans le cas de malversations perverses et autres emplois frauduleux que des compositeurs ou producteurs/arrangeurs en mal de modernité à bas prix n'hésitent pas à faire, sans culture verticale (en profondeur) du son diffusé par hautparleur, avec la suffisance qui sied aux béotiens parvenus.

Cela étant dit, un art simplement (...pas purement...) "haut-parlant», sans ajouts de modes d'expressions visuelles ou gestuelles, continue d'être porteur de mille aventures possibles dès que cette nature de musique électroacoustique s'inscrit, selon moi, dans le large cadre des musiques "aurales», transmises et revendiquées en ce sens, demandant la maîtrise de la mémorisation de traces (humaine ou mécaniquel plutôt que celle d'un langage de traces, d'une écriture dirait Robert Escarpit ${ }^{6}$.

Par trace, j'entends donc tout type d'impression (un vestige) qui laisse dans le temps quelque chose derrière elle, en excluant une écriture codée; soit impression réelle comme les traces d'un sanglier dans le bois ou les perturbations magnétiques sur une bande, un ruban $38 \mathrm{~cm} / \mathrm{s}$, soit impression virtuelle comme le souvenir de sa première montre ou la mémoire d'un air traditionnel appris de routine.

Mais là encore je ne suis pas certain que les défenseurs d'une musique électroacoustique pure (à l'image des mêmes, version instrumentale) placent la pureté au bon endroit, ce qui pourrait entraîner peutêtre une vision en partie pessimiste de l'interrogation : "[...] à la croisée des chemins? », vraie pour les uns comme pour les autres.

Autrement dit, la musique électroacoustique dans sa perspective restreinte Igenre de musique contemporainel est peut-être "à la croisée des chemins" ou, moins irrémédiable, un chemin parmi d'autres usant de l'électricité pour se tracer, mais le phénomène électroacoustique dans sa généralité, objet de ma distinction préalable n'est pas à cette croisée, parce qu'il n'est pas à considérer comme un chemin. C'est un outil d'orientation nouveau surgissant en milieu de siècle pour conquérir de nouveaux territoires audibles, comme la boussole l'a permis en son - très lointain - temps pour les espaces géographiques.
6. Escarpit, R., L'écrit et la communication, coll. «Que sais-je», Presses universitaires de France.

\section{L'électroacoustique, représentation du monde audible}

Ne sous-estimons pas le bouleversement produit, dans l'histoire de nos modes de perception du monde sensible, par la captation du son et sa diffusion différée, persistante. 
Par ce qu'on appellera globalement l'enregistrement, le monde audible va devenir au XX' siècle, et seulement là, à mon sens, représentable.

Quel retard avec le monde visible qui, du fond des grottes de nos lointains ancêtres, est resté tracé, présent à nos yeux! Le monde visible d'alors n'a pas qu'été objectivé par la fixation de traces gestuelles sur un support matériel persistant, mais aussi subjectivé dans le même temps : par le regard porté sur le cheval, par interprétation qu'il en donne, notre ancêtre s'a-perçoit que le monde n'est pas que ce qu'il croyait percevoir.

L'a-perception comme face artistique de la perception pourraiton dire, face énonciatrice; donc non pas privation (a-) mais, expression, "une autre façon de...»?

Par la fixation de traces, l'homme introduit de façon indélébile la conscience d'une différence entre lui et le monde ; il crée un décalage que l'on peut dire poétique, ce que j'appelle un intervalle entre le monde usuel/collectif et son homologue individuel imaginé, manifestant de façon tangible la dimension originale, profonde, illimitée, de chaque homme dans sa relation au monde extérieur.

Mais pour le monde audible, qu'en estil?

Menons une sorte de réflexion expérimentale et comparative, sans certitudes ni références cognitives préalables, par l'observation de trois situations simples et simplifiées : celle où un regardant est face à un objet visuel, peinture ou sculpture par exemple; celle d'un écoutant face à un objet sonore instrumental en train d'être joué (une partition musicale interprétée) ; et celle d'un autre, face à un objet sonore électroacoustique (musique ou phonographie inscrite sur CDI diffusé par les hautparleurs de son salon. Plaçons la notion de représentation au carrefour de ces situations, posons la question du morphème présent de la re-présent-ation dans le temps imparti à chaque situation.

Dans le présent de la contemplation d'un objet visuel fixé et stable, le regardant est la présence vivante de la situation, il est d'une certaine façon l'interprète temporel principal de celle-ci, ne serait-ce que parce qu'il peut aller et venir dans/autour de l'objet, s'arrêter de regarder, reprendre, etc. II a une grande marge de manœuvre par rapport à la représentation. Le temps présent de la situation est très directement lié au regardant, il en a la maîtrise; d'une certaine façon la matière visuelle ignore son propre temps, ce qui n'est pas le cas de la matière sonore qui l'impose.

En effet, prenons le cas de la partition musicale : dans le présent d'une écoute en direct d'un objet instrumental, c'est l'interprète qui est la première présence interprétante de la situation, lui-même asservi à la durée relativement fixée par la partition ${ }^{7}$. L'écoutant n'est que la deuxième présence vivante de cette situation, sa marge de manœuvre par rapport à la représentation proposée est plus faible; il est plus ou moins à la merci de l'interprète : moins si c'est la partition qui l'obsède, beaucoup si c'est l'interprète qu'il adule.
7. La partition du temps d'une musique écrite qu'il ne faut jamais confondre avec le partage du temps des musiques improvisées non idiomatiques en appui sur un contexte et non sur un texte; autre sujet.. 
Dans le présent d'une écoute électroacoustique familiale, dont on ne peut négliger l'importance si l'on en croit la profusion éditoriale purement électroacoustique, la situation semble participer des deux précédentes. D'un côté, l'écoutant redevient l'unique présence vivante de celle-ci, face à un objet fixé et stabilisé dont il peut interrompre et reprendre l'écoute à sa guise; il peut manœuvrer l'objet CD, jour et nuit, d'une façon impensable avec un interprète vivant. D'un autre côté, sa maîtrise temporelle de la situation n'est pas aussi large que celle qu'il a dans le cas d'un objet visuel fixe; si l'on évoque la vitesse de lecture d'un individu face à un objet visuel, l'œil-capteur qui lit est bien celui du regardant. Son œil peut être virtuose, vivace dans ses zoom avant ou arrière, ou bien méticuleux dans ses ralentis, ses retours et ses arrêts sur image, quoi qu'il en soit, il n'y a pas transformation de l'objet, on le parcourt plus ou moins vite ${ }^{8}$.

Mais dans le cas d'un objet audible soumis à une temporalité (scandée/périodique ou matérielle/morphologiquel, l'oreille n'a pas la gestion de la vitesse de lecture; on ne peut pas écouter plus ou moins vite dix secondes de musique ou de discours enregistré, ni revenir sur ses pas sans discontinuité, ni encore s'arrêter, car il y a alors disparition ou transformation ou transmutation de l'objet audible. On ne parcourt pas un objet audible parce qu'il est le parcours temporel lui-même : on devrait, plutôt que d'objet, parler de trajet audible?

Interrogeons-nous donc sur la nature des traces fixées, visuelles ou audibles.

Le peintre fixe, par des traces plus ou moins indélébiles et définitives, ses sensations du moment présent de cette fixation; il fixe des traces de sensations momentanées qui se présentaient à lui dans telle ou telle situation. II fixe définitivement l'EFFET du moment présent vécu par lui ; mais on doit alors parler, maintenant pour nous, d'un ancien moment présent. C'est ce que nous appellerons, en un seul mot, une représentation, particulière, du monde visible d'alors.

Je ne développerai pas ici ce que recouvre le terme sensation, sauf pour dire qu'il est surtout porteur de sens plutôt que de sensiblerie, comme une sorte d'instant ultime et directionnel d'avant le dernier geste fixateur, et qu'il est par là porteur et chargé de toute la conception antérieure, intuitive ou non, qu'il s'agisse de construction ou de bâtissage. Seule certitude, c'est que ce dernier geste est le bon.

Le compositeur instrumental fixe lui aussi des sensations momentanées mais non par des traces représentant directement ces sensations mais par une écriture codée, un langage de traces intermédiaire : la partition. On pourrait dire qu'il fixe des CAUSES opérationnelles et suffisamment significatives permettant de retrouver ultérieurement l'EFFET d'un moment présent vécu. Mais dans ce cas-ci, la représentation particulière du monde audible du compositeur, qui va se faire dans un temps différé, n'est plus de la même nature, car seules les CAUSES ont été fixées définitivement. On ne peut plus parler de fixation des EFFETS d'un moment présent puisque ce qui va être le véritable moment présent vécu par l'écoutant/spectateur, c'est l'interprétation par un instrumentiste particulier des CAUSES fixées et que,
8. J'évite par simplification annoncée le cas de l'objet visuel animé.

9. C'est ce qui est devenu paradoxalement le propre de la révolution électroacoustique dans la production d'art audible; le compositeur électroacoustique demandant à la tête de lecture du magnétophone de jover à l'œil, de tenir le rôle de l'œil (vif, paresseux, pointilleux ou capricieux) lors de ses manipulations de la bande magnétique. Cela a été l'introduction fondamentale et pratique à la matérialité du son que le $X X$ siècle $a$, dès son amorce, suggéré de diverses façons et progressivement généré au gré des trouvailles techniques ou prémonitions diverses (se rapporter aux écrits de Varèse en 1916). Ce fut la naissance de nouvelles façons de faire/laisser des traces et de les fixer, ef l'occasion de rappeler mon refus théorique $\mathrm{d}^{\prime}$ inscrire historiquement la musique électroacoustique comme une branche/fille naturelle de la musique instrumentale écrite. 
contexte oblige, la partition qu'un interprète joue un certain jour à Tombouctou ne produira pas les mêmes EFFETS un autre jour à Los Angeles. Fautil alors, au risque de s'y perdre, parler d'un nouveau moment présent qui se distinguerait de l'ancien moment présent du peintre? Je propose pour l'heure le terme de re-présentation; re-présentation du monde audible, dès lors que ce dernier est causé, provoqué par une écriture, un code, cas d'une partie, et seulement d'une partie, de la musique utilisant les instruments pour son effectuation.

Pour l'électroacoustique en général (document, phonographie, musique), la fixation de traces est, comme pour le domaine visuel, définitive. C'est l'EFFET d'un moment présent qui est conservé puis diffusé, qu'il s'agisse d'un reportage en direct, subtil et pertinent, effectué par un homme de radio talentueux lors du couronnement d'une reine quelconque, ou bien de la fixation d'un son de trompette ayant subi trois nuits complètes de transmutations audio-numériques par un compositeur tenace et exigeant. Ce serait de nouveau un ancien moment présent : au moment de la diffusion, il y a représentation, en un seul mot, des traces fixées.

Là encore les choses ne sont pas si simples si, quittant la généralité électroacoustique on s'en prend à la (?) musique électroacoustique et aux multiples chemins qu'elle a empruntés en long, en large et en travers depuis le milieu du $X X^{e}$ siècle $^{10}$.

$\|$ faut bien admettre, si l'on évoque les différentes pratiques (catégories) répertoriées, que la musique électroacoustique selon son orientation est devenue soit une représentation soit une re-présentation du monde audible; qu'elle peut ne pas être que des EFFETS (sans connotation péjorative, on m'aura bien compris) diffusés " tels que", comme un tableau est montré "tel que", mais qu'une part de CAUSES à ces EFFETS soit aussi présente au moment de la diffusion. Car il y a eu très vite comme une envie supplémentaire à la simple écoute aveugle, à la contemplation extatique du sonore. Comme si pour la contemplation d'un tableau, exagérons le raccourci, on ne pouvait se passer du peintre en train de générer ses traces.

On pourrait expliquer de cette façon le développement conjoint, bien que de nature totalement opposée (historiquement, culturellement, économiquement), $d^{\prime}$ une part des outils spécifiques de diffusion en concert ${ }^{11}$, et d'autre part de celui de la musique mixte. Si l'on veut aller vite, je réduirai cette ébauche de réflexion à un souci global d'importer un peu de CAUSES dans les EFFETS par le biais d'une interprétation en direct (console de diffusion ou instrument), c'est-à-dire du nouveau (moment présent) dans de l'ancien. II ne me semble pas utile de débattre de ces soucis et d'argumenter en faveur, ou contre ce que certains vont appeler des prothèses, d'autres des prolongements naturels. Les choses sont, c'est leur immense mérite, gage d'une vitalité intime et complexe de l'aventure face aux atermoiements frileux et circonspects, trop souvent, de certaines autorités de tutelle ou des spécialistes de comptoir. Poursuivons.

Cette volonté d'une dimension interprétante manifestée par les professionnels de la musique électroacoustique en développant soit l'outil de diffusion en concert
10. Encore une fois pas de croisée des chemins ou une éventuelle et pessimiste crucifixion, mais plutôt une gigantesque gare de triage avec personne à la commande... et ça marche!

11. Je renvoie au très argumenté Actes III Composition et diffusion en musique électroacoustique de l'Académie internationale de musique électroacoustique de Bourges, Mnémosyne, 1999. 
(tendance latine), soit la cohabitation instrumentale (tendance anglo-saxonne) n'est pas la seule réponse à ce besoin d'une sorte de marge de manœuvre que l'on souhaiterait large par rapport à un trajet audible fixé : marge de manœuvre suggérant l'envie de poser la main sur l'œuvre, en jouer ou s'en jouer, rendre l'œuvre mobile. Car en se plaçant à un niveau moins fonctionnel et du point de vue de l'écoutant, j'essaierai de montrer que la fixation de traces audibles et la diffusion de celles-ci sans adjuvants techniques d'interprétation ne fige pas la situation présente et que la qualité des sensations momentanées évoquées en début de communication sont plus affaire de relation de chacun au contenu que de réception formelle de catégories de musique. l'écoutant est mobile vis-à-vis de l'œuvre.

Mais avant de quitter cette approche comparative tentant de situer l'électroacoustique par rapport au domaine visuel ou au domaine instrumental écrit, je compléterai le jeu des distinctions théoriques entre représentation et re-présentation par ce qui me semble participer de la présentation. Dans la perspective phonoculturelle proposée, cela concernerait tout ce qui peut se rattacher à une pratique de pure auralité, sans appui sur un "texte opérationnel " qui indique la qualité et quantité de son à produire, comme l'improvisation libre non idiomatique déjà évoquée. C'est le domaine du "je-ici-maintenant» s'exprimant par la voix, l'instrument ou la live electronic dans une relation im-médiate à un contexte et à celuità seul : en un autre lieu (topicité), en un autre temps (chronicité), pour d'autres personnes (socialité), la musique ne peut être la même; sorte de garantie ou de sceau d'authenticité " aurale »'

Ce que l'on admettra, c'est qu'il n'y a pas dans cette nouvelle situation d'effecfuation de musique, le rattachement à de l'ancien, objectivé, comme dans la situation de l'interprétation d'une partition du XVIII' siècle. Plus rien de fixé Itraces ou langage de traces), c'est le contexte lui-même qui devient la part stabilisée de la situation de réalisation musicale ; il n'y a, objectivement, que du présent auquel se rattacher. Le monde audible est présenté.
12.... à relativiser quand on analyse le style d'un improvisateur : s'il y a style, c'est qu'il y a permanence d'une certaine façon de faire, reconnue et partagée par les écoutants. Ne pas confondre invention, c'est-àdire le fait de dé-couvrir quelque chose, et création : une pure genèse, étymologiquement originale.

\section{Musique inscrite électroacoustique : impérative ou infinitive?}

Dans ces derniers propos, mon souhait serait d'exprimer combien me semble vaste le champ d'expérimentation de l'électroacoustique en dehors, en amont plutôt, des recherches sur et autour des moyens de diffusion ou de mise en relation avec d'autres techniques d'expression (instrumentales, visuelles, gestuelles), ou bien en amont des rencontres inter-culturelles favorisant l'hybridité dont on a dit par ailleurs la nécessité régénérante. 
Quittant donc une approche catégorielle de l'électroacoustique, faisons la révision en profondeur de nos propos du point de vue d'un écoutant de trajets audibles (fixation de traces sonores) diffusés par haut-parleurs seuls, sans adjuvants d'aucune sorte, hors contexte spectaculaire et/ou public. Nous sommes alors obligés de nous préoccuper du contenu de ces traces fixées, ce que j'ai jusqu'à maintenant évité dans mes propos.

Constatons l'extrême diversité des traces sonores fixables que ce soit par captation microphonique (de la monophonie à l'halophonie) ou par synthèse de toute nature. Elles vont, aux deux extrêmes, être perçues par notre écoutant soit comme faisant partie de son propre monde audible /reconnaissance immédiate de quelque chose faisant référence), soit comme hors de ses usages perceptifs, comme traces originales, inouïes : référentialité et originalité comme pôles de notre entendement.

Entre ces deux simplifications du monde audible propre à chacun, se place bien sûr toute une multitude de traces sonores intermédiaires due soit à des traitements du son lui-même le rendant ambigu, troublant sa lisibilité, soit à un agencement original d'unités significatives reconnaissables, une par une, mais pas dans cet agencement qui serait hors des usages courants de notre écoutant. Mais la différenciation sera forcément relative, fonction de la culture sonore de l'écoutant, de sa/ses mémoire(s), de ses intentions, de son environnement, du contexte (topicitéchronicité-socialité du son).

Par exemple, l'enregistrement d'une forte pluie insérée dans une émission de radio ou dans une composition électroacoustique ne va pas générer le même EFFET chez un auditeur d'une région tempérée (une simple anecdote) que chez un éleveur de bovin d'une région ovest-africaine en voie de désertification (un espoir vitall. Autre exemple : si j'insère l'enregistrement de glissements d'accords de neuvième de dominante joués au piano dans une composition électroacoustique et que je fais entendre cette composition à une assemblée fortement éprise de Debussy, cette référence va produire du sens supplémentaire à l'écoute, alors que ce même passage, pour une assemblée de pygmées Ba-Benzélé, va être perçu comme inovï ou anecdotique ou comme non significatif : un non-sens.

Gaston Bachelard dans sa Poétique de l'espace ${ }^{13}$ souligne d'un point de vue phénoménologique la différence entre la résonance et le retentissement d'une image poétique. J'interpréterais cette différence de la façon suivante : la résonance consisterait à retenir de l'image un effet horizontal, une résonance de surface, qui ne ferait que prolonger une sensation momentanée; par contre, la dimension verticale (en profondeur) du retentissement toucherait chez l'individu à la fois des zones de connaissances/reconnaissances significatives et des zones d'affects, de sensations lointaines, enfouies, diffuses. On pourrait dire qu'il y a absence d'intervalle entre la CAUSE et l'EFFET dans le cas de la résonance (CAUSE et EFFET sont superposés), alors que le retentissement serait dû à une distance appréciable entre
13. Bachelard, G. (1994) [1957], La poétique de l'espace, coll. « Bibliothèque de philosophie contemporaine $\gg$ Paris, Quadrige/PUF, $216 \mathrm{p}$. 
la CAUSE et l'EFFET. Je précisais cette différence de nature dans un article sur "Le temps en musique électroacoustique ${ }^{14}$ »: "[...] tout son diffusé par un haut-parleur cause un certain effet perceptif et que, en conséquence : a) dans le cas d'une matérialité temporelle originale l'effet devient la cause d'une nouvelle expérience ; et b) dans le cas d'une matérialité temporelle référentielle on a déjà, de l'effet, l'expérience de sa cause".

Rattachons nos propos présents aux deux conséquences $a$ et $b$ évoquées alors :

a) une trace originale (un son inovii) est à l'instant de son écoute un ajout à notre expérience du sonore, elle ne renvoie pas à de l'ancien moment vécu et ne peut donc être entendue que pour ce qu'elle est à l'instant de notre perception : substance, forme ou matière que je vais dire impérative, car on ne peut guère y échapper sauf à se boucher les oreilles. C'est l'EFFET lle son brut effectué) qui est perçu initialement, dans une sorte de nudité originelle in-nommable qui s'impose d'un seul bloc à l'écoutant. Trace in-nommable parce qu'on ne peut pas la rattacher à une expérience passée, du moins à la première écoute. Trace impérative au sens où elle donne d'une certaine façon une réponse avant que se soit posée une question.

Mais cette impérativité n'est pas inéluctable, car dans le cas d'une œuvre électroacoustique que l'on va supposer purement originale, fantasme du créateur-dieu, le compositeur peut utiliser subtilement le phénomène de redondance qui va créer progressivement de l'expérience, du vécu, de ces formes ou matières nouvelles, I'EFFET devenant reconnaissable au cours de l'écoute, devenant peu à peu une CAUSE nommable lau moins une image mentale), permettant progressivement à l'écoutant d'être pour une part l'interprète du moment vécu. II perçoit progressivement un style let y adhère plus ou moins) puisqu'il acquiert progressivement de l'expérience de la substance. Une composition électroacoustique supposée purement originale, que je définirai comme impérative, peut donc, par la durée et l'agencement adéquat, passer au statut de musique inscrite infinitive, c'est-à-dire interprétable, conjugable théoriquement à l'infini par tout écoutant par essence changeant, mobile dans le temps et l'espace de sa vie.

b) une trace de référence, contrairement à la trace originale, renvoie à une expérience passée, mémorisée et plus ou moins pertinente dans notre fonctionnement mental et sensible. Attrait plus ou moins grand selon l'intérêt du moment et la familiarité de l'écoutant avec ces traces sonores, qu'elles soient culturelles, usuelles, fonctionnelles, etc. Mais dès la perception de l'EFFET, une CAUSE répertoriée - la masse d'expérience accumulée concernant cette trace sonore - va surgir et créer une sorte d'intervalle de comparaison entre le passé et le présent, agrémentant d'une façon ou d'une autre la perception. La trace sonore n'est plus entendue "pour ce quelle est " car son histoire dans notre mémoire vient s'interposer, nous donnant la possibilité dans
14. Actes V - Académie de Bourges, Mnémosyne, 1999-2000 
cet intervalle créé de man-œuvrer, de poser la main sur l'œuvre, bref d'interpréter le moment proposé. On peut donc conjuguer ce moment de différentes façons, selon l'heure ou l'âge ou le contexte de réception de ces traces sonores. Un espace de liberté a été créé par ce retentissement variable, interrogatif, autorisant donc des interprétations et des réponses nombreuses; on parlera de musique inscrite infinitive.

Prenons pour exemple une expérience vécue, celle de la diffusion de la phonographie d'une machine à fabriquer de la dentelle " machine à tulle" de la ville de Calais). Dans le cas d'une assemblée de compositeurs internationaux, le son de la machine est perçu dans le meilleur des cas comme un matériau intéressant relativement original, faisant un certain EFFET, exploitable, pourquoi pas. Dans le cas d'une diffusion de la même phonographie à une personne depuis dix ans à la retraite mais ayant travaillé plus de quarante ans sur cette machine, ce sont des larmes d'émotion qui vont commenter cette écoute.

En langage populaire, on entend encore l'expression "ça me CAUSE... " pour "cela me dit bien, cela me touche..." ; c'était fondamentalement le cas pour cette ouvrière "fulliste" qui au-delà de et à travers la trace sonore voyait sa vie défiler, subissait un bouleversement de son moment présent. II y avait production d'é-motion, CAUSÉE par un moment sonore de référence (retentissant) pour elle, mais à peine original pour des spécialistes des sons fixés.

Alors, en schématisant les qualificatifs, s'agissait-il d'une "musique anecdotique" comme on disait (comme on médisait...) il n'y a pas si longtemps, ou d'une "musique dramatique»?

\section{Brève coda sans conclusions réelles}

Un tel exemple, multipliable, me fait donc redouter ce que pourrait être une approche catégorielle (et rassurante?) des musiques électroacoustiques, qui aurait pour but la comptabilisation des chemins et orientations à succès ou à échecs probables ou possibles, présents ou à venir, toute cette démarche effectuée qui plus est par un acteur/inducteur de cette substance, forcément partial.

$\mathrm{Si}$, prudemment, on se place d'une part du large point de vue phonoculturel proposé, où l'auralité, la matérialité et la contextualité du son s'entrecroisent pour discourir sur le sonore en général (et, les incluant, sur des musiques particulières), et d'autre part du point de vue de l'écoutant et de son contexte face à la référentialité et l'originalité relatives des traces fixées, avec ces deux "béquilles" donc, I'horizon électroacoustique me semble encore bien dégagé pour qui aurait gardé le goût de ce que je nommerai une inventure musicale. 
Les réflexions expérimentales (pour voir, comme au poker...) autour de la représentation du monde audible ainsi qu'autour des couples notionnels référentialité/originalité et CAUSE/EFFET me semblent encore partiellement étanches les unes aux autres par manque de recul et de mise à l'épreuve temporelle des arguments; mais même si lesdits arguments devaient "partir en fumée" selon l'expression populaire, selon un dicton tout aussi populaire : " ll n'y a pas de fumée sans feu. " Le feu de l'énergie expérimentale qui couve en chaque être proche de l'électroacoustique.

\section{Bibliographie}

BACHELARD, G. (1994) [1957], la poétique de l'espace, coll. «Bibliothèque de philosophie contemporaine», Paris, Quadrige/Presses universitaires de France.

ESCARPIT, R. (1993), L'écrit et la communication, coll. «Que saisje», Paris, Presses universitaires de France.

SAVOURET, A. (1999-2000), «le temps phonographique», dans Actes V - le temps en musique électroacoustique, Académie internationale de musique électroacoustique de Bourges, Mnémosyne.

SAVOURET, A. (1999), « Solfège de code ou solfège de l'entendre : quelle formation de l'oreille? », dans le Conservatoire de Paris - Deux cents ans de pédagogie, Buchet/Chastel.

(1997) Actes III - Composition et diffusion en musique électroacoustique, introduction de $G$. Bennett, Académie internationale de musique électroacoustique de Bourges,

Mnémosyne. 


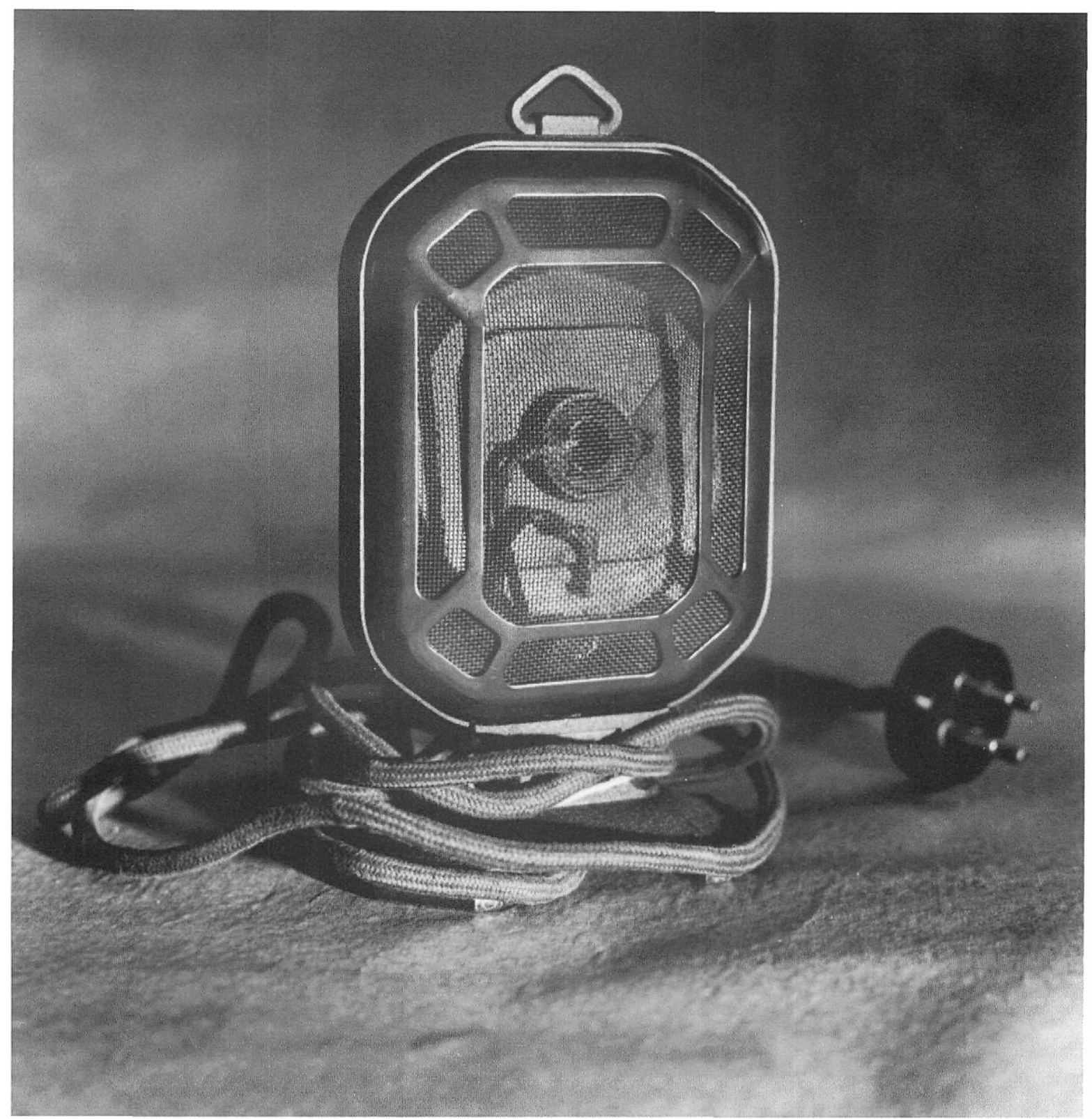

